\title{
Optimal Control of Ankle Joint Moment: Toward Unsupported Standing in Paraplegia
}

\author{
Kenneth J. Hunt, Member, IEEE, Marko Munih, Member, IEEE, Nick Donaldson, and Fiona M. D. Barr
}

\begin{abstract}
This paper considers part of the problem of how to provide unsupported standing for paraplegics by feedback control. In this work our overall objective is to stabilize the subject by stimulation only of his ankle joints while the other joints are braced. Here, we investigate the problem of ankle joint moment control. The ankle plantarflexion muscles are first identified with pseudorandom binary sequence (PRBS) signals, periodic sinusoidal signals, and twitches. The muscle is modeled in Hammerstein form as a static recruitment nonlinearity followed by a linear transfer function. A linear-quadraticGaussian (LQG)-optimal controller design procedure for ankle joint moment was proposed based on the polynomial equation formulation. The approach was verified by experiments in the special Wobbler apparatus with a neurologically intact subject, and these experimental results are reported. The controller structure is formulated in such a way that there are only two scalar design parameters, each of which has a clear physical interpretation. This facilitates fast controller synthesis and tuning in the laboratory environment. Experimental results show the effects of the controller tuning parameters: the control weighting and the observer response time, which determine closed-loop properties. Using these two parameters the tradeoff between disturbance rejection and measurement noise sensitivity can be straightforwardly balanced while maintaining a desired speed of tracking. The experimentally measured reference tracking, disturbance rejection, and noise sensitivity are good and agree with theoretical expectations.
\end{abstract}

Index Terms - Functional electrical stimulation, modelling, optimal control, rehabilitation engineering.

\section{INTRODUCTION}

$\mathbf{P}$ ARALYZED muscles generally retain their ability to contract, and electrical stimulation is used in rehabilitation as a therapy because it restores muscle bulk and brings several collateral benefits [1]. The authors are interested in functional electrical stimulation (FES) in which the aim is to restore paralyzed muscles to some normal motor activities. For people who have spinal cord injuries at the thoracic level, these activities include standing and stepping. Artificial systems which stimulate the motor nerves in order to cause the muscles

Manuscript received March 14, 1997. Recommended by Associate Editor, B. K. Ghosh.

K. J. Hunt is with the Centre for Systems and Control, Department of Mechanical Engineering, Glasgow University, Glasgow G12 8QQ, Scotland (e-mail: k.hunt@mech.gla.ac.uk).

M. Munih is with the Faculty of Electrical Engineering, University of Ljubljana, Ljubljana, Slovenia.

N. Donaldson is with the Department of Medical Physics and Bioengineering, University College London, London, U.K.

F. M. D. Barr is with the Royal National Orthopaedic Hospital Trust, Stanmore, U.K.

Publisher Item Identifier S 0018-9286(98)04156-7. to contract are neuroprostheses since they replace a defective part of the natural neuromuscular system.

The seminal paper in FES was by Kantrowitz [2] who demonstrated that paraplegics could stand with stimulation. Throughout the 35 years since then, the putative advantages of applying feedback control to stimulators have been acknowledged. At present, however, it is little used. There are several reasons for this, one being that, to date, most FES systems have been external, with electrodes attached to the skin over the muscles and with sensors strapped to the joints or limb segments. The concomitant unsightliness and need to make frequent attachments and detachments has probably deterred many from using the systems and certainly has kept the systems simple in order to be practicable. The most common arrangement is to place electrodes over the quadriceps muscle group in the thigh to extend the knees, perhaps over the buttock muscles to extend the hips, and over the peroneal nerves below the knee to cause flexion at all the leg joints by reflex action [3]. Because fatigue of the quadriceps may limit standing time, goniometers have been added at the knees to provide angle feedback signals so that the quadriceps stimulation may be modulated. Ideally the quadriceps contraction should just be sufficient to maintain standing, and therefore the fatigue rate should be less than if there is no feedback, in which case the intensity remains strong [4], [5].

In the same period implant technology has advanced, and we now have stimulators with many channels [6] which we could use with implanted sensors to make more complex systems without the subject having the penalty of a greater setting-up time. Given this possibility, the question of how to control the stimulation is important and is the subject of research at several centers [7]-[9].

From the point of view of the Control Engineer, the physiology of the nerve stimulation, its activation of the muscle, and the mechanical properties of the muscle are mostly well understood [10], [11]. This has usually been the focus of attention when considering feedback control. However, if we consider the mechanics of a paraplegic standing up using stimulation of his knee extensors, we find that, using whatever handles he has to help lift and balance, and his upper-body musculature, he is able to apply moments to his paralyzed leg joints [12]. In this way, the intact neuromuscular system exercises sufficient control to allow most paraplegics to stand. It also means that artificial feedback control of knee angle, as described above, occurs in parallel to the control actions of the brain. It follows that such knee controllers, though they may be perfectly satisfactory for their purpose, have not been 
designed conventionally on the basis of measurements from the plant. The parameters for the controllers have been chosen empirically, partly on the subjective opinion of the paraplegic user.

The paralyzed muscles, seen as the plant, are nonlinear timevarying with a transmission delay and a low-pass frequency characteristic [10]. We would like to know how to control this plant when the intact neuromuscular system cannot interfere; to consider, for example, whether performance can be improved if we can stimulate with irregular interpulse intervals [13]-[15] or make the controller adaptive to compensate for fatigue [16]. The isolated distal spinal cord of a paraplegic continues to exhibit reflex activity which is manifested as spasticity, in which there is coactivation of antagonist muscles resulting in limb rigidity and spasm. We would also like to know how well the controllers cope with the spasticity and spasm manifested in real subjects.

We have chosen to conduct experiments on the ankles and are using a special apparatus called the Wobbler [17]. The ankles have a number of advantages for study: during quiet standing, the joints remain at mid-range; since the masses of the feet are small, the ankle moments can be easily and accurately estimated from external torque transducers aligned with the joints; and the ankle muscles are easily stimulated with electrodes on the calves. Our subjects stand in the apparatus, strapped into a polythene body brace which extends from their head to their shanks immobilizing their other joints. They do not and need not use their hands for support and therefore, in this arrangement, the intact neuromuscular system cannot interfere with the controller at the ankles. The objective is to see whether we can artificially restore balance by stimulation of the ankle muscles, analogous to one mode in which normal people stand. The Wobbler allows a series of tests to be performed which may lead to this objective. In all these tests except "actual standing," the subject is held vertical by stay ropes. Some tests are isometric, in which case the feet (in the foot boxes) are fixed and the torques in the supporting shaft are measured to observe the ankle moments. Alternatively, the stiffness of the ankles can be measured by applying sinusoidal motion at frequencies up to $6 \mathrm{~Hz}$. Controllers can be tested isometrically or in motion, the latter imitating the situation during artificial-balance standing, except that instead of the feet being on the ground and the body swaying, the body is fixed while the feet are rocked.

An early decision [18] was to follow the lead set by Bajzek and Jaeger [19], using a nested controller structure in which the ankle moments (left and right) and the common ankle angle (equivalent to the inclination of the whole body during "actual standing") were fed back so that the whole controller is more robust. The overall control strategy envisaged for ankle angle control is shown in Fig. 1. An inner-loop controller $C_{m}$ provides relatively high bandwidth control of muscle moment, while the outer loop controller $C_{\theta}$ controls the angle. The output of the angle controller is the desired moment set-point.

In our current series of experiments, we are using optimal control methods, and this paper describes the estimation of muscle model parameters, the design of the moment controllers, and shows results from a normal subject.

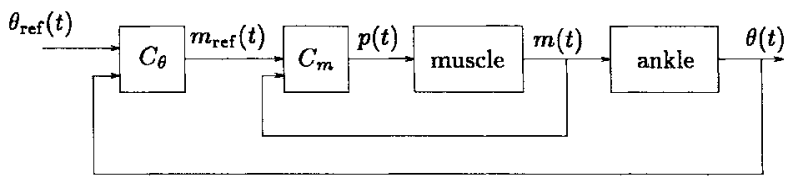

Fig. 1. Cascade control structure for unsupported standing: $\theta$ is measured ankle angle, $m$ is measured ankle moment, $p$ is muscle stimulation pulse width, $\theta_{\text {ref }}$ is angle set-point, $m_{\text {ref }}$ is moment set-point, $C_{\theta}$ is angle controller, and $C_{m}$ is moment controller.

From the control engineer's point of view, a major aim in this work was to provide a controller design setup for straightforward day-to-day use in the laboratory. It is desirable to have a small number of controller design parameters, each of which have a clear physical interpretation and which allow direct tuning and tradeoff of the key closed-loop properties: reference tracking, disturbance rejection, and measurement noise sensitivity. This aim was achieved by specializing the polynomial equation approach for the linear-quadratic-Gaussian (LQG) regulator design to the problem at hand. The polynomial approach [20], [21] provides a very clear transfer-function alternative to the Wiener-Hopf [22] and state-space [23] versions of the LQG regulator. Efficient numerical algorithms are available for controller computation [20], [24].

Moment-controller results from a paraplegic subject are reported in [25] and [26]. The development to the nested controller for angle allowing actual balanced standing has also been carried out [27], [28].

Notation: This work employs real polynomials in one indeterminate $q^{-1}$, where $q^{-1}$ is to be interpreted as the unit delay operator described by the relation $q^{-1} x(t)=x(t-1)$ for a sequence $x(t)$. For simplicity, the arguments of polynomials are sometimes omitted such that polynomial $X\left(q^{-1}\right)$ is denoted as $X$. For any polynomial $X\left(q^{-1}\right)$ its adjoint in positive powers of $q$ is denoted by $X^{*}\left(q^{-1}\right)=X(q)$.

\section{CONTROLlER SYNTHESIS AND ANALYSIS}

\section{A. Plant Structure and Optimal Controller}

The feedback control structure for muscle moment is depicted in Fig. 2. The main signals in this structure are as follows.

$m(t) \quad$ Muscle moment.

$m_{\text {ref }}(t)$ Desired moment (reference).

$a(t) \quad$ Desired muscle activation.

$p(t) \quad$ Stimulation pulse width.

$d(t) \quad$ Disturbance generation signal.

$n(t) \quad$ Measurement noise.

The signal $u(t)$ is a notional control signal associated with the idealized setup of Appendix 1. The disturbance and measurement noise signals $d$ and $n$ are modeled as mutually uncorrelated stochastic signals with intensities $\psi_{d}$ and $\psi_{n}$, respectively.

The muscle is modeled in Hammerstein form and consists of the static recruitment nonlinearity $f_{r}$ followed by the linear transfer function $\frac{q^{-k} B_{m}\left(q^{-1}\right)}{A_{m}\left(q^{-1}\right)}$. Procedures for experimental determination of $f_{r}, A_{m}$, and $B_{m}$ are described in Section V. The integer $k \geq 1$ is a discrete input-output time delay. $A_{m}$ 


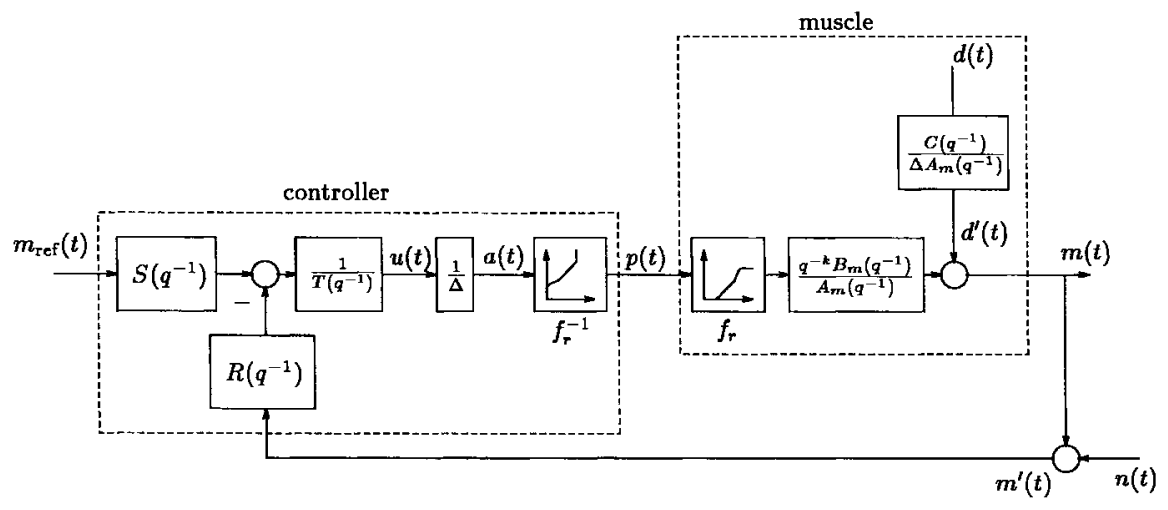

Fig. 2. Closed-loop system for muscle moment, $\Delta=1-q^{-1}$.

and $B_{m}$ are polynomials in the delay operator $q^{-1}$ and have the form

$$
\begin{aligned}
& A_{m}\left(q^{-1}\right)=1+a_{1} q^{-1}+\cdots+a_{n a} q^{-n a} \\
& B_{m}\left(q^{-1}\right)=b_{0}+b_{1} q^{-1}+\cdots+b_{n b} q^{-n b} .
\end{aligned}
$$

The net effect of disturbances is represented at the output by the signal $d$ driving the filter $\frac{C\left(q^{-1}\right)}{\left(1-q^{-1}\right) A_{m}\left(q^{-1}\right)}$. The integrating term $\Delta=1-q^{-1}$ is included here to model the effect of stepwise-changing constant disturbances and offsets, which are typical due to the muscle physiology. ${ }^{1}$ In addition to model error and other effects, a major source of disturbance is muscle stiffness [17]. Rejection of muscle stiffness disturbances is experimentally verified in the sequel.

The recruitment nonlinearity is cancelled by an inverse nonlinearity $f_{r}^{-1}$ at the controller output. Assuming perfect cancellation the open-loop muscle can be represented by the model

$$
m(t)=\frac{q^{-k} B_{m}\left(q^{-1}\right)}{A_{m}\left(q^{-1}\right)} a(t)+\frac{C\left(q^{-1}\right)}{\left(1-q^{-1}\right) A_{m}\left(q^{-1}\right)} d(t) .
$$

Due to the difficulty in obtaining reliable models of the noise polynomial $C$, it is assumed in the sequel that $C=1$.

The moment is corrupted by a measurement noise $n$ so that the signal

$$
m^{\prime}(t)=m(t)+n(t)
$$

is available for feedback. Since the muscle contains no inherent integrating behavior, and since the disturbance contains constant offsets, an integrator must be incorporated in the controller. This is represented by the term $\frac{1}{\Delta}$, with $\Delta=$ $1-q^{-1}$, in Fig. 2. The controller has a two-degrees-of-freedom form so that the reference tracking behavior can be influenced independently of the feedback loop properties and is described by

$$
\begin{aligned}
a(t) & =\frac{1}{\left(1-q^{-1}\right)} u(t) \\
& =\frac{1}{\left(1-q^{-1}\right) T\left(q^{-1}\right)}\left(S\left(q^{-1}\right) m_{\mathrm{ref}}(t)-R\left(q^{-1}\right) m^{\prime}(t)\right)
\end{aligned}
$$

\footnotetext{
${ }^{1}$ Such nonstationary disturbances can be represented in the stochastic framework of Appendix 1 by considering $d$ to be a compound or generalized Poisson process, i.e., a sequence consisting of random pulses of variable magnitude occurring at random times; see [21] for details.
}

where $S, R$, and $T$ are polynomials in the delay operator. The choice of $S$ is described in Section III. The polynomials $T$ and $R$ are determined in the optimization procedure and correspond to the solution of the optimal regulator problem.

The optimization cost function is chosen to allow a simple tradeoff between output moment variance and control variable activity. A suitable cost function to achieve this aim is

$$
J=\epsilon\left[m^{2}(t)+\rho\left(\left(1-q^{-1}\right) a\right)^{2}(t)\right]
$$

where $\epsilon$ is the expectation operator and $\rho>0$ is the tunable control weighting. Due to inclusion of integral action via the $\Delta$ term, it is in fact the increments in activation $a$ which are penalized. A formal solution to this optimization problem, including underlying assumptions and solvability conditions, is given in Appendix 1. Here, the solution is specialized to the muscle moment control setup of Fig 2. The controller polynomials $T$ and $R$ which correspond to the minimal value of the cost-function (6) are obtained from the solution of the linear polynomial equation

$$
\left(1-q^{-1}\right) A_{m} T+q^{-k} B_{m} R=D_{f} D_{c}
$$

subject to the condition

$$
\left(\left(1-q^{-1}\right) A_{m}\right)^{-1} R \text { strictly proper }
$$

i.e., $\operatorname{deg}(R)<\operatorname{deg}\left(A_{m}\right)+1$. A sufficient condition to ensure existence of a unique solution with this property is that the polynomials $A_{m}$ and $B_{m}$ have no common factors ${ }^{2}$ (see Appendix 1).

The polynomials $D_{c}$ and $D_{f}$ in (7) are the stable solutions to the spectral factorizations

$$
\begin{aligned}
& D_{c} D_{c}^{*}=B_{m} B_{m}^{*}+\left(1-q^{-1}\right) A_{m} \rho A_{m}^{*}(1-q) \\
& D_{f} D_{f}^{*}=\psi_{d}+\left(1-q^{-1}\right) A_{m} \psi_{n} A_{m}^{*}(1-q) .
\end{aligned}
$$

Stable solutions for $D_{c}$ and $D_{f}$ exist when $A_{m}$ and $B_{m}$ have no unstable common factors.

\footnotetext{
${ }^{2}$ This condition can be easily checked numerically. If stable common factors are detected, the two coupled equations, (46) and (47), can be solved.
} 


\section{B. Feedback Loop Analysis}

By some straightforward algebraic manipulation, the closedloop equations relating the external signals $m_{\text {ref }}, d^{\prime}$ and $n$ to the signals $m$ and $a$ are obtained

$$
\begin{aligned}
m(t)= & \frac{q^{-k} B_{m} S}{A T+q^{-k} B_{m} R} m_{\mathrm{ref}}(t)+\frac{A T}{A T+q^{-k} B_{m} R} d^{\prime}(t) \\
& -\frac{q^{-k} B_{m} R}{A T+q^{-k} B_{m} R} n(t) \\
a(t)= & \frac{A_{m} S}{A T+q^{-k} B_{m} R} m_{\mathrm{ref}}(t)-\frac{A_{m} R}{A T+q^{-k} B_{m} R} d^{\prime}(t) \\
& -\frac{A_{m} R}{A T+q^{-k} B_{m} R} n(t) .
\end{aligned}
$$

Here, $A=\left(1-q^{-1}\right) A_{m}$.

A number of the above transfer functions play an important role in the frequency-domain design and analysis of the controllers. The effect of disturbances on the output is determined by the sensitivity function, denoted $\tilde{S}$, and from (11) defined by

$$
\tilde{S}=\frac{A T}{A T+q^{-k} B_{m} R} .
$$

Also important is the effect of measurement noise which is determined by the complementary sensitivity function $\tilde{T}$

$$
\tilde{T}=\frac{q^{-k} B_{m} R}{A T+q^{-k} B_{m} R} .
$$

Note from (13) and (14) that these functions are related by $\tilde{T}=$ $1-\tilde{S}$, and this is the reason for the term "complementary." This reveals the fundamental tradeoff between disturbance rejection and measurement noise effects: to reject disturbances $\tilde{S}$ should ideally be "small," and to make the output insensitive to measurement noise $\tilde{T}$ should also be small. Due to the relation $\tilde{T}=1-\tilde{S}$, this is clearly not possible. A solution may be provided in specific cases by making $\tilde{S}$ small in frequency ranges where disturbances occur (usually low frequencies) while letting it grow at frequencies where measurement noise is known to predominate (normally high frequencies). A further important point is to look at the effect of disturbances and noise on the control signal $a$. From (12) it is clear that both affect the control signal through the transfer function

$$
\left(\frac{q^{-k} B_{m}}{A_{m}}\right)^{-1} \tilde{T}
$$

This again implies that ideally $\tilde{T}$ should be kept small, but the frequency characteristics of the plant $B_{m} / A_{m}$ (which is low pass) also need to be considered here. The sensitivity and complementary sensitivity functions will be used in the sequel to analyze the feedback properties of the muscle moment control loop for various design parameter settings.

\section{Practical Aspects And Design Procedure}

\section{A. Effect of Design Parameters}

The parameters which affect the calculation of the optimal controller are the control weighting $\rho$ and the intensities of the disturbance and measurement noise signals $\psi_{d}$ and $\psi_{n}$. Since these intensities are difficult to determine accurately, they can be considered, along with $\rho$, as control design parameters. Since from (10) it is merely the relative values of $\psi_{d}$ and $\psi_{n}$ which determine $D_{f}$, it may be assumed without loss of generality that $\psi_{d}=1$. Thus, there are two scalar control design parameters: control weighting $\rho$ and measurement noise intensity $\psi_{n}$.

The effect of varying $\rho$ can be seen clearly from the cost-function definition (6). Higher values of $\rho$ will penalize changes in activation more strongly, and the variance of the muscle moment will be correspondingly higher. In frequency domain terms, higher $\rho$ will reduce the bandwidth of the closed loop, resulting in poorer disturbance rejection but less sensitivity to measurement noise. The qualitative effect of changes in $\rho$ on reference tracking is the same, i.e., faster reference tracking is achieved with lower control weighting. The direct effect of $\rho$ on the sensitivity and complementary sensitivity functions will be illustrated in the sequel for specific muscle parameters.

The effect of measurement noise intensity $\psi_{n}$ can be seen from the filter spectral factorization (10) for $D_{f}$. It should be noted that the filter spectral factor corresponds to the observer poles in a state-space formulation of the regulator problem (see [29] and [30]). As $\psi_{n} \rightarrow 0$, the feedback system effectively has a deadbeat observer since $D_{f} \rightarrow 1$ in this case. On the other hand, as $\psi_{n}$ tends toward a large number, the observer poles tend to the poles of the open-loop system, which in the case of the ankle muscle has low-pass characteristics. The effect of increasing the noise intensity is thus to generate faster roll-off of the loop transfer function. This will reduce sensitivity to measurement noise (for the control signal in particular) while generally leading to somewhat poorer disturbance rejection. As described in Section III-B, the controller setup is such that $\psi_{n}$ has no effect on tracking speed.

An alternative method for choice of the observer poles which avoids the spectral factorization (10) is based on the observation that increasing $\psi_{n}$ leads to a low-pass characteristic with a dominant pair of poles (the muscle transfer function is predominantly second-order). The alternative is therefore to directly specify the observer poles to correspond to a secondorder transfer function with given time-domain properties, such as rise-time and damping. This method has been successfully implemented for moment control. It is assumed that the desired damping factor is unity (critical damping) and only the rise-time, denoted $t_{\mathrm{obs}}$, must be specified. This specification of rise-time and unity damping leads directly to an equivalent continuous-time transfer function

$$
\frac{\omega_{n}^{2}}{s^{2}+2 \omega_{n} s+\omega_{n}^{2}}
$$

where $\omega_{n}$ is the natural frequency associated with specified rise-time $t_{\mathrm{obs}}$ and unity damping factor. From a simple timedomain analysis of the second-order system (16), $\omega_{n}$ can be shown to be related to $t_{\mathrm{obs}}$ through $\omega_{n}=3.2 / t_{\mathrm{obs}}$. $D_{f}$ is then given by the denominator of the discretized version of this transfer function. Qualitatively, increasing $t_{\mathrm{obs}}$ has the same effect as increasing $\psi_{n}$. The effects of varying the design parameters are summarized in Table I. 


\section{B. Reference Tracking}

Servo performance is defined by the transfer function between $m_{\text {ref }}$ and $m$, denoted as $G_{m / m_{\text {ref }}}$, and from (11) and (7) is given by

$$
G_{m / m_{\mathrm{ref}}}=\frac{q^{-k} B_{m} S}{A T+q^{-k} B_{m} R}=\frac{q^{-k} B_{m} S}{D_{c} D_{f}} .
$$

The open-loop zeros appear in this expression since in general the optimal controller performs no zero cancellation within the loop. ${ }^{3}$ It is important to introduce the reference signal in such a way that it does not excite the observer dynamics [30]. This is achieved by constraining $S$ as

$$
S\left(q^{-1}\right)=\lambda D_{f}\left(q^{-1}\right)
$$

where $\lambda$ is a scalar which is set to achieve unity steady-state gain in this closed-loop transfer function. Combining (17) and (18), the closed-loop transfer function becomes

$$
G_{m / m_{\mathrm{ref}}}=\frac{\lambda q^{-k} B_{m}}{D_{c}}
$$

and the appropriate value of $\lambda$ is therefore

$$
\lambda=D_{c}(1) / B_{m}(1)
$$

The reference tracking properties of the system as defined by $G_{m / m_{\text {ref }}}$ are effectively decoupled from measurement noise effects due to the constraint (18). Thus, the design variable $\psi_{n}$ (or $t_{\text {obs }}$ ) has no effect on the speed of reference tracking; servo characteristics depend solely on the control weighting $\rho$. The lower $\rho$ is, the faster reference tracking is. ${ }^{4}$ On the other hand, perturbation of the output from the setpoint will be affected by changes in $\psi_{n}$ (or $t_{\mathrm{obs}}$ ) because the effect of disturbances and measurement noise will change; generally, when $\psi_{n}$ increases the disturbance rejection will be poorer, but the sensitivity to measurement noise will improve (see Table I).

\section{Design Procedure}

The design procedure can be summarized in the following steps:

Given data: $A_{m}, B_{m}, k$. Choose design parameters: $\rho, t_{\mathrm{obs}}$. Step 1) Perform spectral factorization

$$
D_{c} D_{c}^{*}=B_{m} B_{m}^{*}+\left(1-q^{-1}\right) A_{m} \rho A_{m}^{*}(1-q)
$$

for stable $D_{c}$.

Step 2) Convert the given $t_{\mathrm{obs}}$ to corresponding natural frequency $\omega_{n}=3.2 / t_{\mathrm{olos}}$, and calculate $D_{f}$ as the denominator of the discretized transfer function $\frac{\omega_{n}^{2}}{s^{2}+2 \omega_{n} s+\omega_{n}^{2}}$.

\footnotetext{
${ }^{3}$ Cancellation of stable zeros could be arranged if desired by incorporating the zeros to be cancelled as factors of $T$.

${ }^{4}$ The tracking response can additionally be decoupled from the disturbance rejection response through the introduction of a reference prefilter. Since such a filter appears outside the feedback loop, the closed-loop dynamics can be arbitrarily shaped by cancelling $D_{c}$ and the stable part of $B_{m}$ [see (19)] and introducing the desired closed-loop reference transfer function. In the experiments reported here, satisfactory performance was achieved without this additional measure.
}

TABLE I

Effect of Varying Controller Design Parameters

\begin{tabular}{c|ccc}
\hline & $\begin{array}{c}\text { tracking } \\
\text { speed }\end{array}$ & $\begin{array}{c}\text { disturbance } \\
\text { rejection }\end{array}$ & $\begin{array}{c}\text { noise } \\
\text { sensitivity }\end{array}$ \\
\hline$\rho \uparrow$ & slower & worse & better \\
$\rho \downarrow$ & faster & better & worse \\
$\psi_{n}, t_{\text {obs }} \uparrow$ & no change & worse & better \\
$\psi_{n}, t_{\text {obs }} \downarrow$ & no change & better & worse \\
& & & \\
\hline
\end{tabular}

Step 3) Calculate $\lambda$

$$
\lambda=D_{c}(1) / B_{m}(1)
$$

Step 4) Calculate $S$

$$
S\left(q^{-1}\right)=\lambda D_{f}\left(q^{-1}\right)
$$

Step 5) Solve the equation

$$
\left(1-q^{-1}\right) A_{m} T+q^{-k} B_{m} R=D_{f} D_{c}
$$

for $T\left(q^{-1}\right)$ and $R\left(q^{-1}\right)$, subject to $\operatorname{deg}(R)<$ $\operatorname{deg}\left(A_{m}\right)+1$

Step 6) Evaluate sensitivity functions and closed-loop performance. If necessary, choose new design parameters following the guidelines of Section III-A and return to Step 1).

\section{WobBler SETUP AND EXPERIMENTAL CONDITIONS}

Disregarding various safety features, the Wobbler comprises two "foot boxes," in which the subject's shoes are fixed with the ankles aligned to the supporting shaft, which can be put in sinusoidal motion by a crank mechanism driven from a DC motor. Two torque transducers measure the ankle moments and these are resolved by the A/D card in the PC486 to $0.1 \mathrm{Nm}$ quantization steps; a shaft encoder measures the angle in increments of 0.018 degrees. There is also a tachometer on the DC motor which is connected to another A/D input. The computer is connected by a serial port to a "Stanmore Stimulator" [31], operating in "Laboratory Mode."

The software for the experiments comprises a library of $\mathrm{C}$ programs. Beside the digital controller algorithm, there are many other tasks, including:

1) instructing the stimulator to deliver pulses and checking the transmission $(20-\mathrm{Hz}$ pulse frequency);

2) reading the $A / D$ inputs at $20 \mathrm{~Hz}$;

3) reading the shaft encoder at $20 \mathrm{~Hz}$;

4) storing variables in RAM during each test;

5) saving variables to .BIN files on disc at the end of each test;

6) calculating the stimulus pulse width from the current activation level using the inverse recruitment function. 
There are also several utilities, used later for converting the .BIN files to ASCII format. These .DAT files are then loaded by the appropriate MATLAB script which plots and analyzes the results or may design a controller using given design parameters.

Before a session of experiments, 2-in-diameter self-adhesive electrodes are attached on the midlines of the soleus and gastrocnemius muscles of the subject. A neurologically intact subject can step into the apparatus before the body brace is strapped on and the stays tightened to maintain a normal upright posture with the feet horizontal. While standing quietly, the A/D inputs are read as well as the torque readings used for the zero ankle moment values during subsequent tests.

Stimulus intensity is a function of both the pulse width and the pulse current, both of which are adjustable in the Stanmore Stimulator. For these experiments, we use pulse width to modulate the intensity, having set the current initially in Test A. This is done by choosing a starting current [10, $20,30 \cdots 150] \mathrm{mA}$ and then ramping up the pulse width in a $20-\mathrm{Hz}$ train while measuring the ankle moment. Usually the response is sigmoidal. In a normal subject, this is repeated at increasing currents until it begins to become painful, usually at $60 \mathrm{~mA}$. The maximal moment with a maximal pulse width of $500 \mu$ s is typically 50 to $60 \mathrm{Nm}$ in one leg. This nearly painful current is adopted for the following tests. The pulse width is defined in steps of $2 \mu \mathrm{s}$ in the range $0-500 \mu \mathrm{s}$.

One method of measuring the frequency response is by Fourier transformation of the twitch responses, the responses to well-separated stimulus pulses from which the muscle contraction or twitch is completed before the next stimulus pulse. As Durfee and Maclean [32] pointed out, this test has two advantages: it causes little fatigue and it also finds the recruitment curve for the muscle. In our Test $B, 50$ pulses are applied at 1.2-s intervals; they are of ten pulse widths and in random order. The five responses for each pulse width are averaged, and the results are plotted as a surface with moment versus pulse width and time since the last pulse. The peak value of each averaged twitch, plotted versus the stimulus pulse width, is the recruitment curve for that channel. Having found the inverse recruitment curve, we then describe the level of stimulation by its "activation level," which is the input to the inverse recruitment block $(a(t)$ in Fig. 2). In our experiments we do not use Fourier methods to determine the nonparametric frequency responses. Instead, parameter identification methods are used with a variety of open-loop activation test signals [sine and pseudorandom binary sequence (PRBS)] to estimate the linear transfer-function part of the muscle response (see Section V-B).

After designing one or more controllers, Test $M$ is of closedloop reference tracking. The usual reference signal is a step up, onto which is then superimposed a sine wave with three cycles at each of $[0.3,0.5,0.8,1.2,2,3.2] \mathrm{Hz}$ frequencies, followed by a step down to zero. The same control program is used to observe the disturbance rejection except that the reference is a step up to a level which is maintained during most of the test, then a step down. While the reference is constant, the feet are rocked so that the ankle moments are disturbed due to the ankle stiffnesses. In the following results, we make step changes to

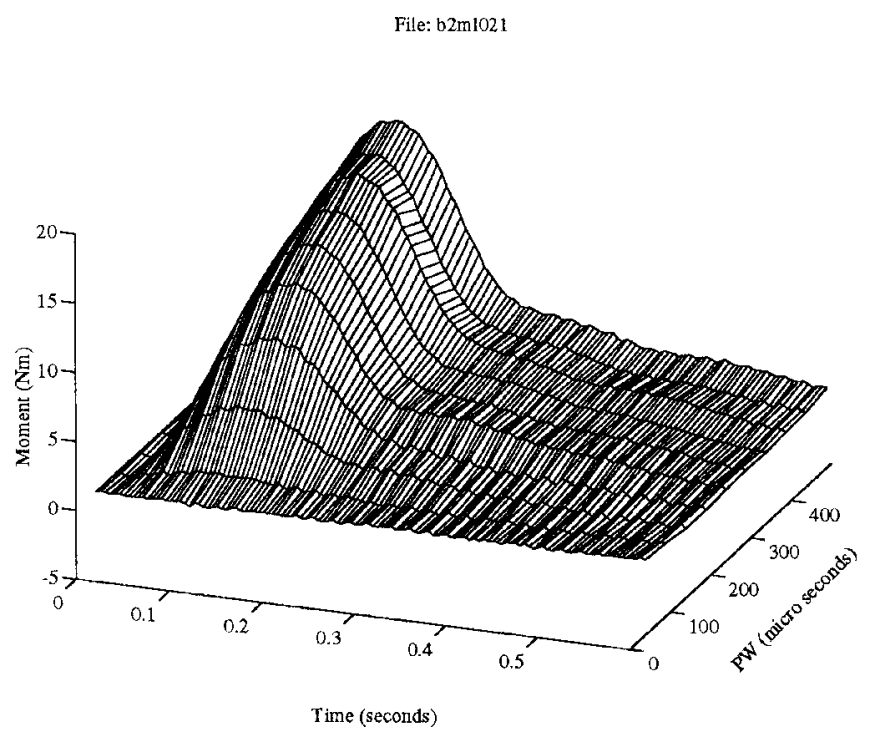

Fig. 3. Twitch response data for estimation of recruitment nonlinearity.

the frequency of rocking during this test. Experimental results for reference tracking and disturbance rejection are given in Section VI.

The disturbance rejection test cannot be evaluated on its own without a measure of the ankle stiffnesses. This is done in Test $H$. While the ankles are rocked at one of the rocking frequencies used $([0.3,0.5,0.8,1.0] \mathrm{Hz})$, the muscle is activated for 3 cycles with $a(t)=[0,200,400,600,800,1000]$ mAct. The test is repeated for all the rocking frequencies.

\section{Muscle Modelling: ExPerimental Results}

\section{A. Approximation of Recruitment Nonlinearity}

Test $B$, as described above, involves the application of isolated pulses with 10 distinct pulse widths of $[50,100, \ldots$ 500] microseconds. A typical set of averaged responses is shown in Fig. 3, which shows measured ankle moment against time, for the range of pulse widths applied.

The recruitment nonlinearity is obtained by plotting the maximum moment for each pulse width, against the pulse width. For each pulse width this results in a notional muscle activation $\tilde{a}$, normalized in the range $0-1$ Act. It is assumed that a pulse width of $500 \mu$ s corresponds to maximum activation, i.e., to $1000 \mathrm{mAct}$. Thus, to find the activation $\tilde{a}^{p}$ (in mAct) corresponding to pulse width $p$ we use

$$
\tilde{a}^{p}=\frac{m_{\max }^{p}}{m_{\max }^{500}} \cdot 1000
$$

where $m_{\max }^{500}$ is the maximum moment measured for $p=500$ and $m_{\max }^{p}$ is the maximum moment measured for the given pulse width $p$. An approximation to the recruitment curve is obtained by interpolating the experimental points, and the recruitment nonlinearity is denoted as

$$
\tilde{a}=f_{r}(p) .
$$




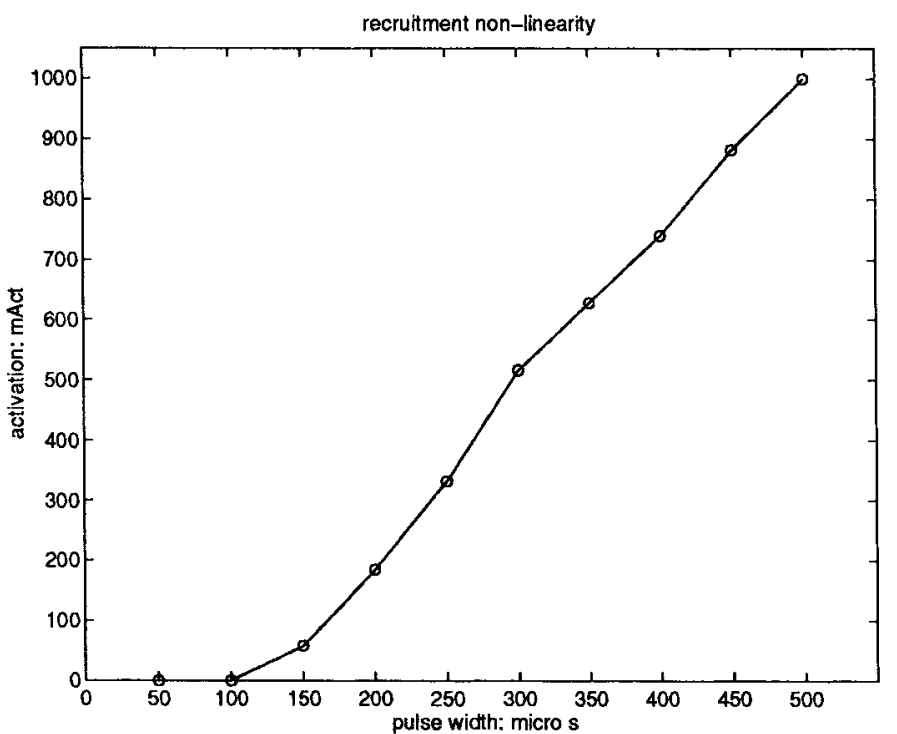

Fig. 4. Typical recruitment curve $f_{r}$.

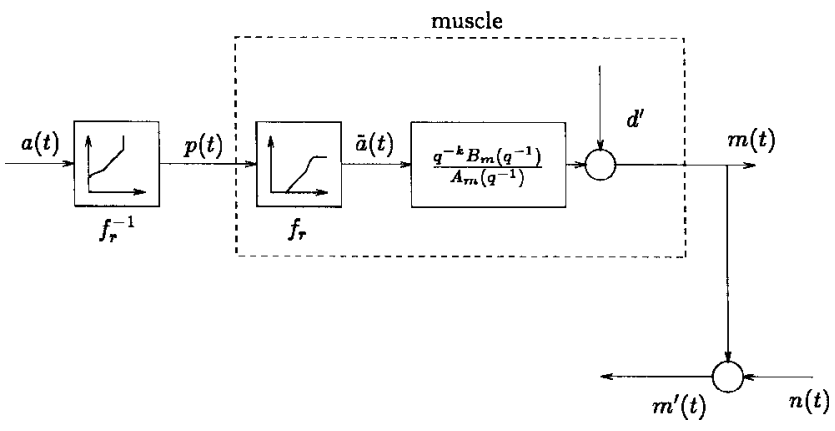

Fig. 5. Open-loop system for muscle identification, $d^{\prime}=d / A_{m}(1)$.

For some desired muscle activation $a$ the estimated pulse width required is given by the inverse recruitment nonlinearity

$$
p=f_{r}^{-1}(a) \text {. }
$$

A typical recruitment curve obtained using this method is shown in Fig. 4.

\section{B. Estimation of Transfer Function}

In order to generate test data for estimation of the linear part of the muscle model, the approximated recruitment nonlinearity is first cancelled; the setup for open-loop identification is shown in Fig. 5.

A series of activation test signals $a(t)$ are applied, and the ankle moment measurement $m^{\prime}(t)$ is generated. For the purposes of estimation it is assumed that the disturbance term $d^{\prime}$ is a constant offset as generated by a pulse $d(t)$ with magnitude $d$. Following application of the final-value theorem for $d^{\prime}(t)$ this leads to an open-loop measurement equation [c.f. (3)]

$$
m^{\prime}(t)=\frac{q^{-k} B_{m}\left(q^{-1}\right)}{A_{m}\left(q^{-1}\right)} a(t)+\frac{1}{A_{m}(1)} d+n(t) .
$$

Assuming the measurement noise $n$ is an uncorrelated zeromean stochastic signal, the best one-step-ahead moment pre- diction is

$$
\hat{m}(t)=\theta^{T} \phi(t-1)
$$

where the parameter vector $\theta$ and regression vector $\phi$ are defined by

$$
\begin{aligned}
\theta= & \left(a_{1} \cdots a_{n a} ; b_{0} \cdots b_{n b} ; d\right)^{T} \\
\phi(t-1)= & \left(-m^{\prime}(t-1) \cdots-m^{\prime}(t-n a)\right. \\
& a(t-k) \cdots a(t-k-n b) ; 1)^{T} .
\end{aligned}
$$

A measure of model fidelity is provided by the least squares criterion

$$
J_{N}(\theta)=\frac{1}{N} \sum_{t=1}^{N}\left(m^{\prime}(t)-\hat{m}(t)\right)^{2}
$$

where $N$ is the number of data points. The analytical solution for the optimal parameter estimate is [33], [34]

$$
\hat{\theta}=\left(\sum_{t=1}^{N} \phi(t-1) \phi^{T}(t-1)\right)^{-1} \cdot\left(\sum_{t=1}^{N} \phi(t-1) m^{\prime}(t)\right) .
$$

This is the solution used in the identification experiments reported in the sequel.

In addition to the twitch responses used for determination of the recruitment nonlinearity, two further types of activation test signal can be employed for transfer-function identification.

1) A sinusoidal signal of variable frequency: A representative test signal and the corresponding moment measurement are shown in Fig. 6(a). A typical model obtained from the sinusoidal activation test signal shown in Fig. 6(a) is

$$
\begin{aligned}
\frac{q^{-k} B_{m}\left(q^{-1}\right)}{A_{m}\left(q^{-1}\right)} & =\frac{q^{-1}\left(0.0073-0.0044 q^{-1}\right)}{1-1.5712 q^{-1}+0.6473 q^{-2}} \\
d & =0.293 .
\end{aligned}
$$

The measured moment and the output predicted by this model with the input of Fig. 6(a) is shown in Fig. 6(b).

2) A PRBS-type activation signal: A representative PRBS test signal and the associated moment measurement are shown in Fig. 7(a). A typical model obtained from the PRBS-type activation test signal shown in Fig. 7(b) is

$$
\begin{aligned}
\frac{q^{-k} B_{m}\left(q^{-1}\right)}{A_{m}\left(q^{-1}\right)} & =\frac{q^{-1}\left(0.0033+0.0027 q^{-1}\right)}{1-1.1525 q^{-1}+0.305 q^{-2}} \\
d & =-0.61 .
\end{aligned}
$$

The measured moment and the output predicted by this model with the input of Fig. 7(a) is shown in Fig. 7(b).

From the twitch responses shown in Fig. 3, the one at a pulse width of $350 \mu$ s was also used for parameter estimation, again with the above least squares criterion. The estimated transfer function in this case was

$$
\frac{q^{-k} B_{m}\left(q^{-1}\right)}{A_{m}\left(q^{-1}\right)}=\frac{q^{-1}\left(0.0047+0.0037 q^{-1}\right)}{1-1.2588 q^{-1}+0.4922 q^{-2}} .
$$

Since for test $B$ the operating point for the muscle is the rest position with $a=m=0$, no offset term was estimated, 


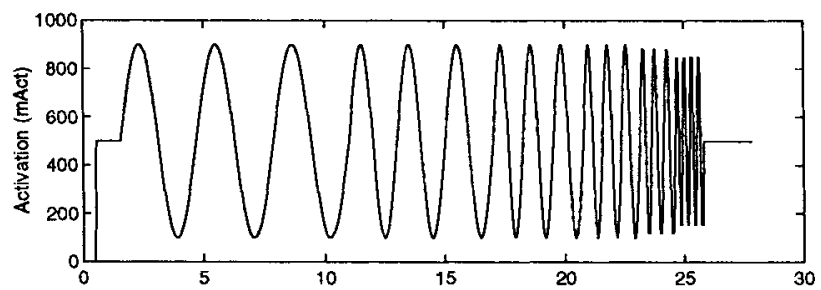

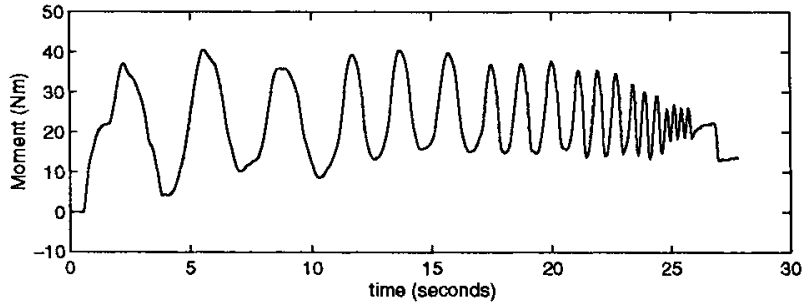

(a)

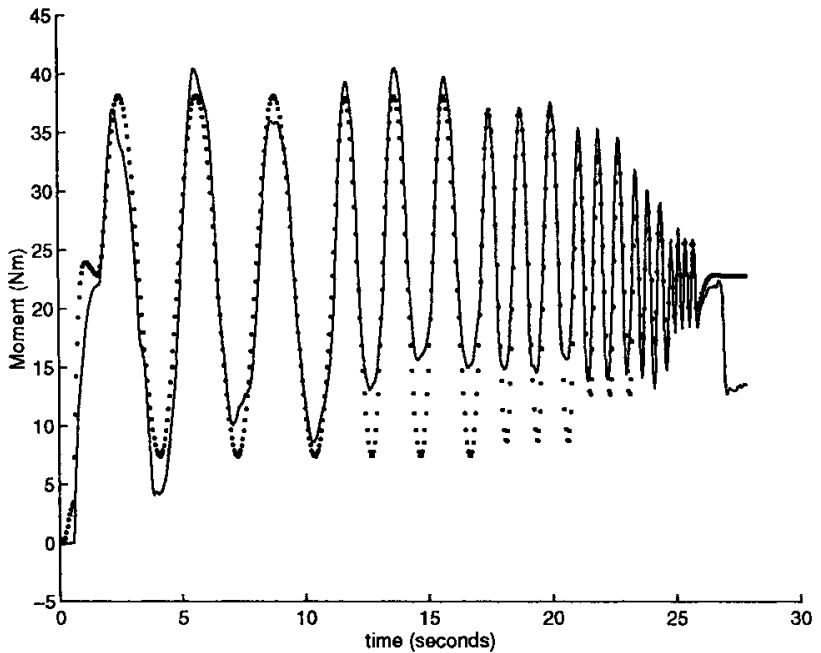

(b)

Fig. 6. Identification with sinusoidal test signal. (a) Activation test signal and measured moment. (b) Measured moment (full line) and model simulation (dotted line).
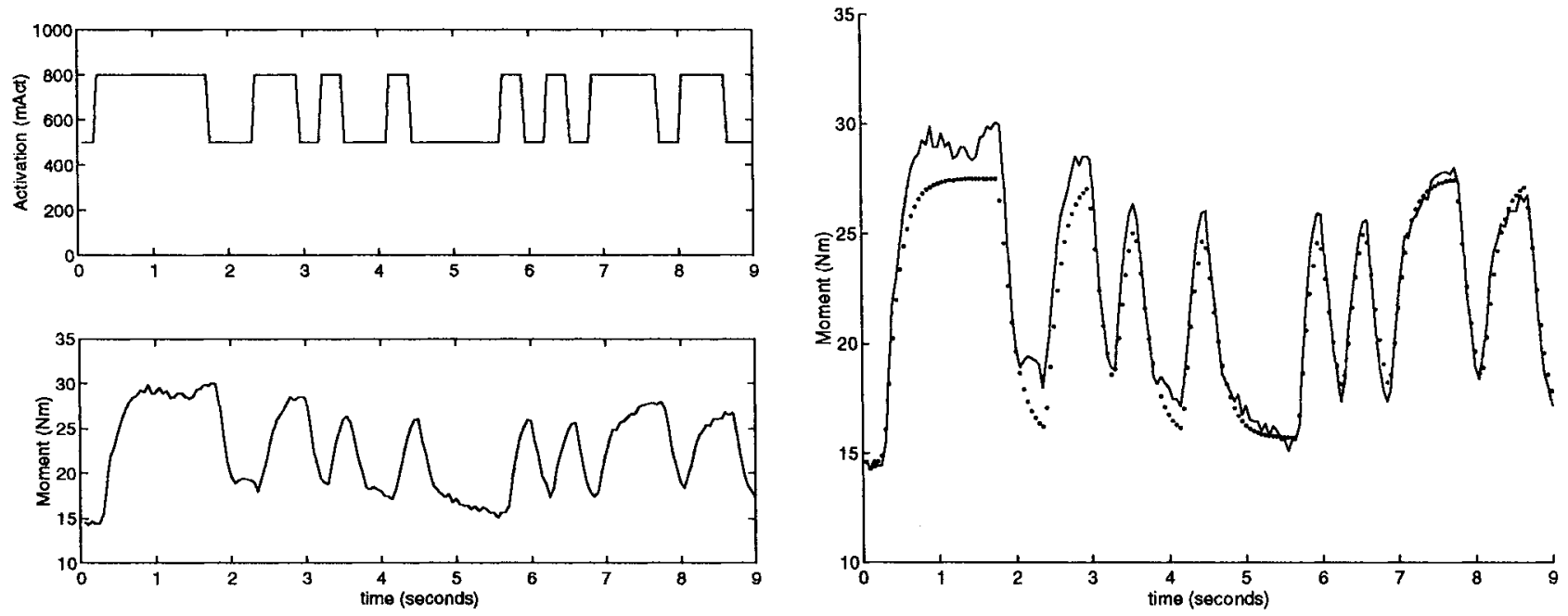

(a)

(b)

Fig. 7. Identification with PRBS test signal. (a) Activation test signal and measured moment. (b) Measured moment (full line) and model simulation (dotted line).

i.e., $d=0$. The model (38) is the one used as the basis for controller design and testing in the sequel.

It should be mentioned that extensive model validation tests using additional data not used for parameter estimation and multiple-step-ahead prediction criteria have confirmed that the second-order model structure with a delay $k=1$ is appropriate for this application. Since new identification tests must be performed very quickly, using as brief a test signal as possible, each time the subject undergoes controller testing, this is done by simply performing one of the tests described above without extensive model validation taking place. The full range of estimation and "off-line" model validation tests will be reported elsewhere [35].

\section{Moment Control: Experimental Results}

Given a pair of estimated muscle polynomials $A_{m}$ and $B_{m}$, the design parameters $\rho$ and $t_{\mathrm{obs}}$ represent tuning knobs for the controller. The calculated controller polynomials $R, S, T$ define its servo tracking performance, disturbance rejection, and the influence of measurement noise. The effects of both tuning knobs on all three types of performance were investigated by simulation and verified by experiments in the Wobbler (Section IV). It will be seen that the qualitative effects of the design parameters, as outlined in Section III-A, are verified by the experimental results. In one measurement session, controllers designed with different $\rho$ and $t_{\text {obs }}$ values were tested in the left leg of the same person and under constant environmental conditions. The control tests were preceded by tests for estimation of the recruitment curve and the linear transfer function parameters (38).

1) To show the effect of varying $\rho$ with a fixed $t_{\mathrm{obs}}=0$ value (deadbeat observer):

a) High value of $\rho=0.5$ : The time-domain response for the reference tracking test is presented in 

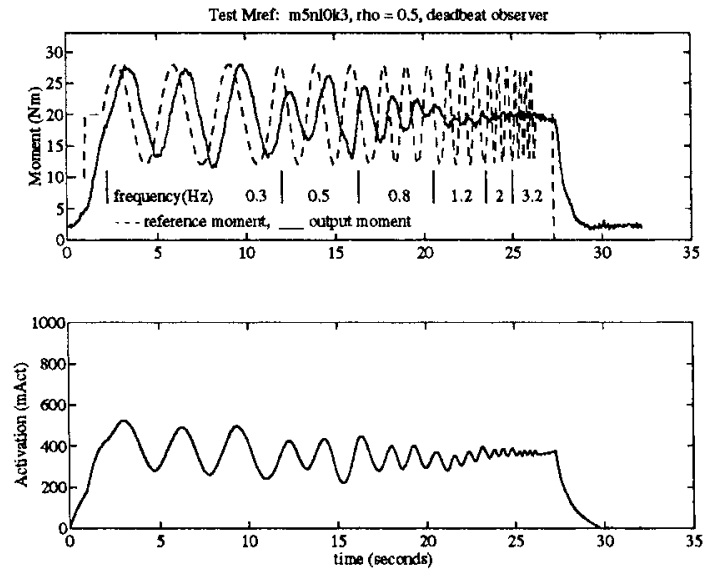

(a)
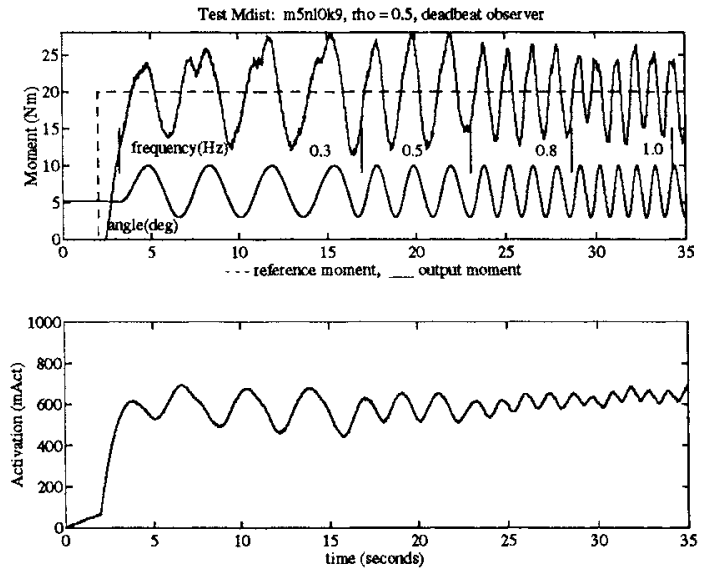

(b)

Fig. 8. Closed-loop control tests: $\rho=0.5, t_{\mathrm{obs}}=0$. (a) Tracking test. (b) Disturbance test: ankle angle perturbed at indicated frequency and magnitude.

Fig. 8(a) with the required reference signal as a dashed curve and output response as a solid curve. The bottom part of the figure shows muscle activation (internal controller variable). The disturbance response is shown similarly in Fig. 8(b).

The reference signal depicted in the upper graph as a dashed line is kept constant, while the feet are rocked with a constant angle amplitude (solid line). The controller output moment, which should ideally be equal to the required moment, is also shown as a solid line. Activation is shown in the bottom part of the figure. In the reference test, poor controller servo tracking is seen with a noticeable phase lag and some amplitude attenuation even at the lower test frequencies. This is expected from the cost-function (6), which, at $\rho=0.5$, strongly penalizes activation changes. That changes in $m$ are not penalized enough is also evident in the negligible disturbance moment rejection.

Ankle stiffness, which directly defines the ankle disturbance moment, is a nonlinear function of the activation, ankle rocking frequency, and ankle angle and was measured in test $H$ as an open-loop disturbance moment (Table II).

The activation level during the disturbance test [Fig. 8(b)] was at approximately $600 \mathrm{mAct}$, and for the first wobbling frequency at $0.3 \mathrm{~Hz}$ the measured disturbance moment is equal to approximately $18 \mathrm{Nm}$ (from Table II). Comparing this with the $18-\mathrm{Nm}$ amplitude in the output moment in the disturbance test, we see no attenuation at the 0.3$\mathrm{Hz}$ wobbling frequency and the same is true at other frequencies. On the other hand, with the high $\rho=0.5$ value, measurement noise effects are small: the reference tracking and disturbance activations are both smooth and noise-free.

b) A low value of $\rho=0.00005$ was selected in contrast to case 1a). The reference tracking results are shown in Fig. 9(a) and the disturbance tests in Fig. 9(b). An excellent amplitude response and very
TABLE II

The Peak-to-Peak Stiffness Moment in NANOMETERS aS a Function of Activation and Wobbling Frequency. StifFness ApPEARS as an OUtPut Disturbance

\begin{tabular}{|c|c|c|c|c|c|c|}
\hline activation (mAct) & 0 & 200 & 400 & 600 & 800 & 1000 \\
\hline freq & & & & & & \\
\hline $0.3 \mathrm{~Hz}$ & 7.6 & 7.7 & 11.2 & 18 & 18 & 19 \\
\hline $0.5 \mathrm{~Hz}$ & 7.1 & 7.2 & 8.4 & 9.8 & 14.5 & 10.3 \\
\hline $0.8 \mathrm{~Hz}$ & 7.0 & 7.5 & 8.5 & 9.1 & 11.8 & 13 \\
\hline $1.0 \mathrm{~Hz}$ & 6.9 & 6.9 & 7.7 & 8.9 & 14.9 & 15 \\
\hline
\end{tabular}

small phase lag appears during reference tracking for signals up to $2 \mathrm{~Hz}$. With the same controller setup, disturbance signals are very well attenuated. But, as a penalty for a wider bandwidth, lower $\rho$ significantly increases measurement noise in the system, which is noticeable from the activation in both the reference tracking and disturbance tests.

Values of $\rho<0.00005$ are for this application practically unusable due to an increasing activation instability, while controllers with $0.00005<\rho<$ 0.5 show effects between those two cases in all three respects: reference tracking, output disturbance rejection, and measurement noise sensitivity.

To interpret the performance changes in the frequency domain, Fig. 12(a) shows the sensitivity and the complementary sensitivity functions (13) and (14) for $\rho=[0.00005,0.005,0.5]$. Low $\rho$ values in the cost function (6) give low phase lag, better disturbance rejection, but poorer noise sensitivity, as the bandwidth is higher for a smaller $\rho$ (changes in bandwidth can be seen in the complementary sensitivity plots). For $\rho=0.5$, on the other hand, the complementary sensitivity function magnitude is low over the frequency range (low noise sensitivity), while the sensitivity function magnitude is large (which allows disturbances to affect the output and control signals). 

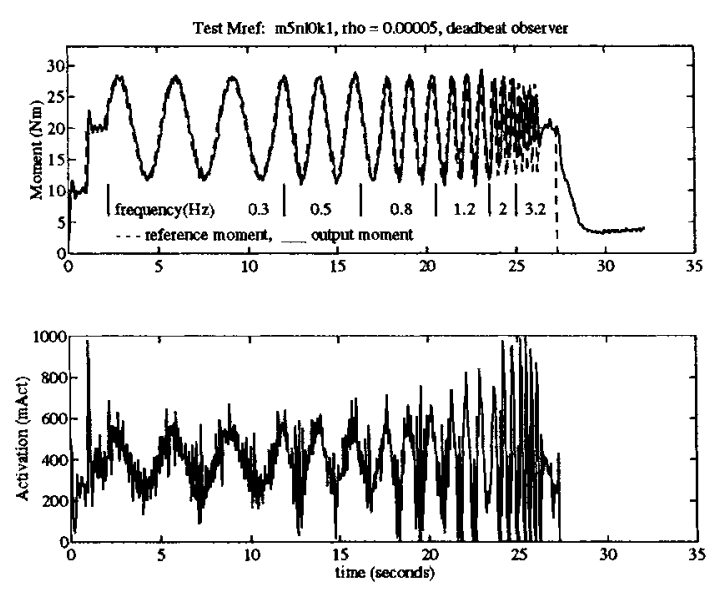

(a)
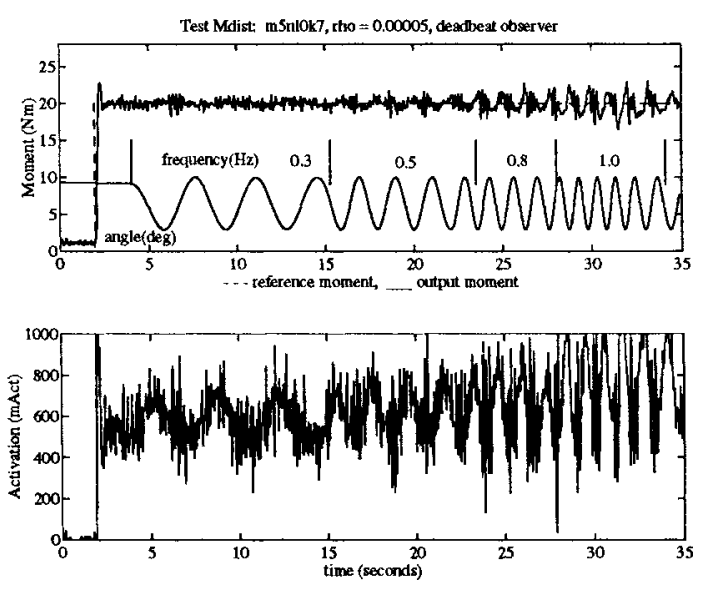

(b)

Fig. 9. Closed-loop control tests: $\rho=0.00005, t_{\text {obs }}=0$. (a) Tracking test. (b) Disturbance test: ankle angle perturbed at indicated frequency and magnitude.
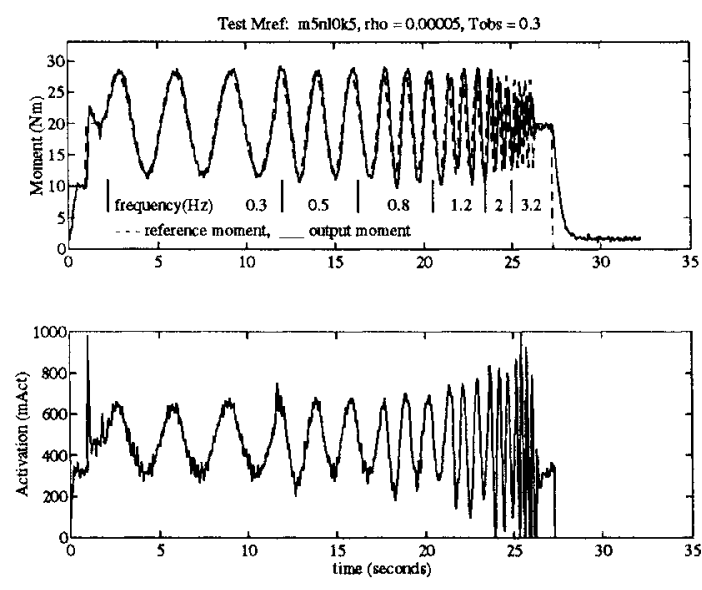

(a)
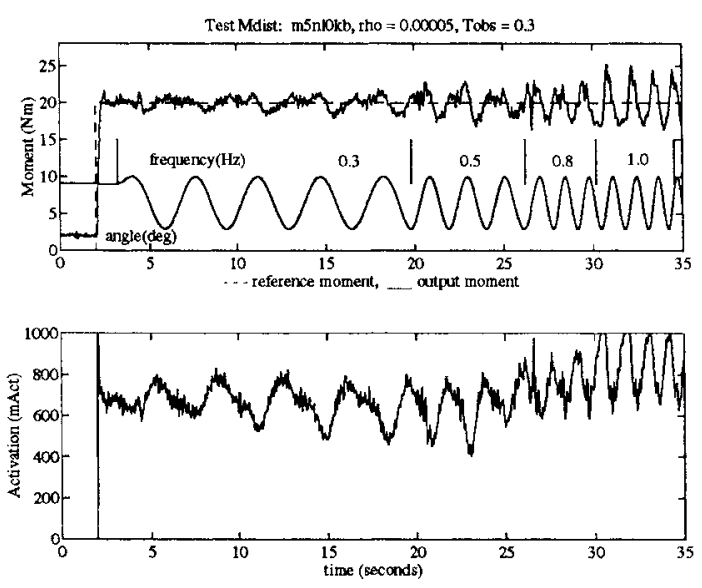

(b)

Fig. 10. Closed-loop control tests: $\rho=0.00005, t_{\text {obs }}=0.3$. (a) Tracking test. (b) Disturbance test: ankle angle perturbed at indicated frequency and magnitude.

2) To show the effect of varying $t_{\text {obs }}$ with a fixed value of $\rho=0.00005$ :

a) Medium value of $t_{\mathrm{obs}}=0.3$ : This $\rho$ value was selected as a good compromise setting with satisfactory tracking speed. The results of reference tracking and disturbance tests are shown in Fig. 10(a) and (b), respectively, and may be compared to Fig. 9(a) and (b), where the $\rho$ value is the same but the observer is deadbeat.

Little difference is seen in the output moment amplitude, phase lag, or superimposed noise responses. But, the noise present in the activation is much smaller than with the deadbeat observer in both the reference and disturbance tests. There is slightly poorer disturbance moment attenuation with this $t_{\mathrm{obs}}$ value.

b) High value of $t_{\mathrm{obs}}=0.8$ : Fig. 11(a) and (b) shows the results of the reference tracking and disturbance tests.
The disturbance test is similar to the poor disturbance response found with $\rho=0.5$, where the injected moments are not attenuated. The reference tracking is still fast with larger $t_{\mathrm{obs}}$ values but is degraded compared to the controllers having lower $t_{\mathrm{obs}}$ or a deadbeat observer due to poor disturbance rejection. The noise in the activation is smaller than for $t_{\mathrm{obs}}=0.3$ but noticeably higher than with large $\rho$ values and deadbeat observers.

The decrease in closed-loop bandwidth for higher $t_{\mathrm{obs}}$ may also be seen from the sensitivity and complementary sensitivity functions which are shown in Fig. 12(b).

\section{CONCLUSION}

Ankle moment controllers are part of the overall controller for unsupported standing (Fig. 1) being tested on paraplegic subjects [27], [28]. These experiments will help to assess 

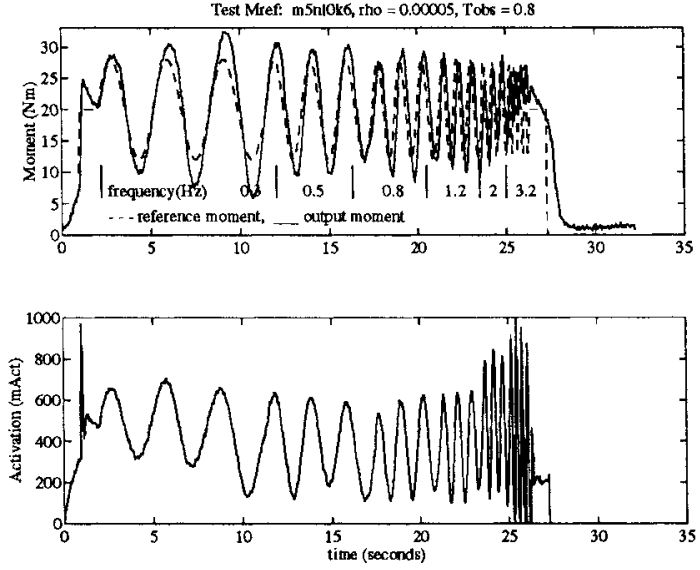

(a)
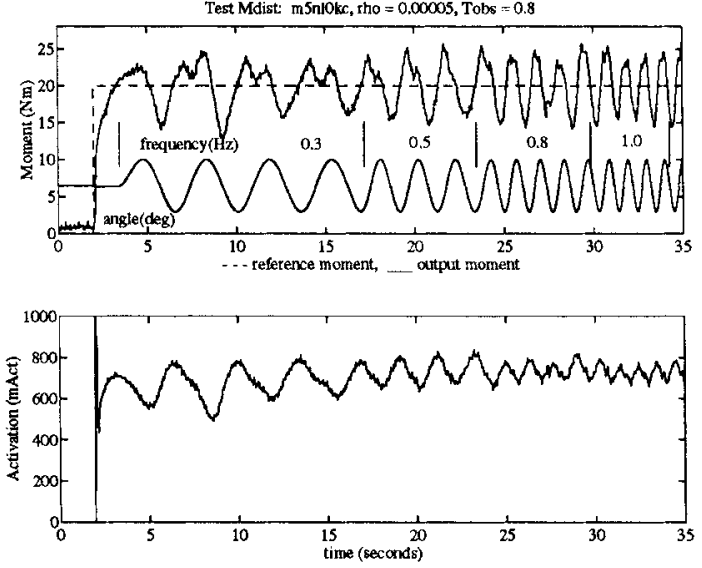

(b)

Fig. 11. Closed-loop control tests: $\rho=0.00005, t_{\mathrm{obs}}=0.8$. (a) Tracking test. (b) Disturbance test: ankle angle perturbed at indicated frequency and magnitude.

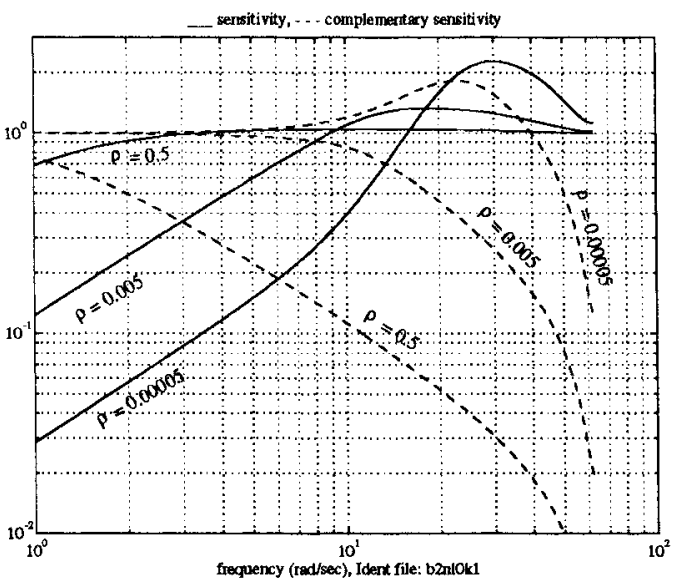

(a)

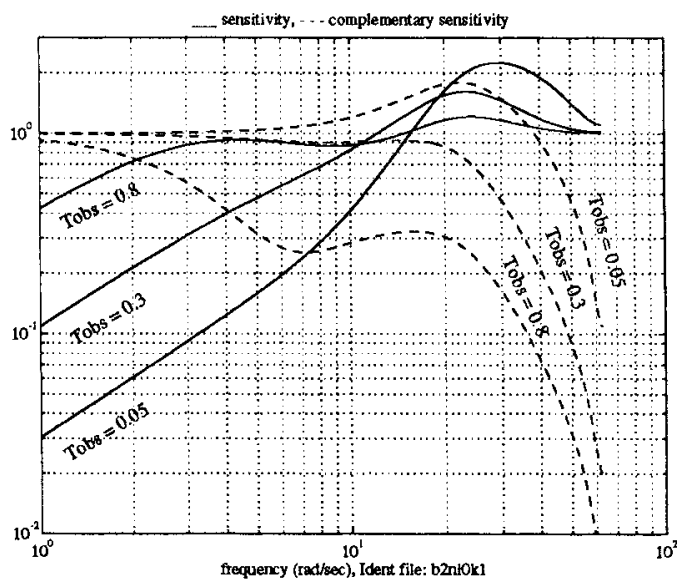

(b)

Fig. 12. Sensitivity and complementary sensitivity functions. (a) Effect of varying $\rho$ with fixed $t_{\mathrm{obs}}=0$. (b) Effect of varying $t_{\mathrm{obs}}=0$ with fixed $\rho=0.00005$

the feasibility of using controller stimulators for unsupported standing, leaving the arms free for manipulative tasks.

In this paper we specialized the theory of optimal control with the polynomial equation approach to ankle joint moment control. The particularly transparent nature of the polynomial formulation resulted in the ability to tune controllers using only two scalar design parameters. The muscle was stimulated at constant stimulus pulse frequency and a Hammerstein model structure, to account for the recruitment nonlinearity, was assumed. Results from a neurologically intact subject in the Wobbler are presented. Results from paraplegics are presented in further papers [25], [26].

If these methods are to pass into clinical practice, an important consideration is the time taken to set up the controllers. After the subject has been helped into the Wobbler and secured in place, the time to determine the stimulator currents and collect the data for the recruitment and frequency responses of the muscles, in both legs, is about $5 \mathrm{~min}$. Then by using MATLAB scripts which minimize the amount of keyboard interaction, controllers with several combinations of $\rho$ and $t_{\mathrm{obs}}$ can be designed in another $2 \mathrm{~min}$, after which closed- loop controller experiments can begin. Demonstrably, this is a reasonably quick procedure, even compared to the often rather short time that paraplegics may comfortably stand. Another favorable observation, for which we present no data here, is that the frequency responses of the muscles change little from session to session, so that it may not be necessary to modify the controllers for each subject very often.

It is clear from these results that there is a very broad range of $\rho$ and $t_{\mathrm{obs}}$ over which the controllers are satisfactory as regards reference tracking, disturbance rejection, and the effect of measurement noise. This range is from $0.00005 \leq \rho \leq$ 0.001 and $0 \leq t_{\mathrm{obs}} \leq 0.4$. The measurement quantization noise in this case is due to the $0.1 \mathrm{Nm}$ resolution of the A/D convertors on the torque load cells which here are measuring moments in the range up to $50 \mathrm{Nm}$.

For the more satisfactory controllers [Fig. 9(a), (b)], we see that the closed-loop bandwidth $(-3 \mathrm{~dB})$ is $15 \mathrm{rad} / \mathrm{s}$, when reference tracking, at which the phase lag is small. Is this adequate? There is no absolute answer to this question: the wider is the bandwidth, the greater the disturbance from which the controller may recover. Possible disturbances are 


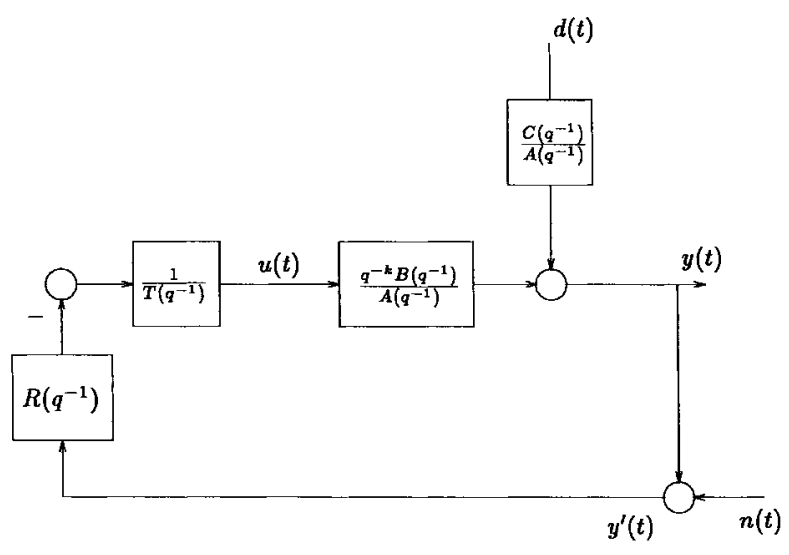

Fig. 13. Closed-loop system—regulator problem.

unpredictable, and some may indeed be too great for recovery however fast the controller; in these cases a normal subject would make a step with one leg rather than be able to recover entirely by exertion of the ankle plantarflexors. Another consideration is of the spectrum of the sway seen in normal subjects; in quiet standing, there is little motion above $1 \mathrm{~Hz}$ [36]. Compared to $1 \mathrm{~Hz}$, our controllers have a bandwidth about 2.5 times greater, which is relatively fast. Because paraplegics are weaker than normals, they will not be able to produce such large accelerations in the inverted pendulum, and so the sway frequencies will be even lower than $1 \mathrm{~Hz}$.

The disturbance rejection is also good. This is less important for stabilizing the inverted pendulum, as the ankle stiffness actually helps stability [17], but it may be relevant to other applications that satisfactory muscle controllers can give both good reference tracking and output disturbance rejection.

\section{APPENDIX 1}

\section{LQG PROBLEM FORMULATION AND SOLUTION}

The general setup for the LQG regulator problem is depicted in Fig. 13.

The open-loop plant is described by

$$
y(t)=\frac{q^{-k} B\left(q^{-1}\right)}{A\left(q^{-1}\right)} u(t)+\frac{C\left(q^{-1}\right)}{A\left(q^{-1}\right)} d(t)
$$

and the measurement equation is

$$
y^{\prime}(t)=y(t)+n(t)
$$

$y$ is the plant output and $u$ is the control signal. The disturbance and measurement noise signals $d$ and $n$ are mutually uncorrelated stochastic signals with intensities $\psi_{d}>0$ and $\psi_{n}>0$, respectively. It is assumed that the following hold.

A1) The subsystem $W_{p}=\frac{q^{-k} B\left(q^{-1}\right)}{A\left(q^{-1}\right)}$ is strictly causal, i.e., $k \geq 1$.

A2) The subsystems $W_{p}=\frac{q^{-k} B\left(q^{-1}\right)}{A\left(q^{-1}\right)}$ and $W_{d}=\frac{C\left(q^{-1}\right)}{A\left(q^{-1}\right)}$ are free of unstable hidden modes.

There is no loss of generality in assuming a common denominator polynomial $A$. $A$ is the lowest common denominator of the subsystems $W_{p}$ and $W_{d}$. A causal regulator having the form

$$
u(t)=-\frac{R\left(q^{-1}\right)}{T\left(q^{-1}\right)} y^{\prime}(t)
$$

is sought. The desired optimal regulator evolves from minimization of the cost function

$$
J=\epsilon\left[y^{2}(t)+\rho u^{2}(t)\right]
$$

where $\epsilon$ is the expectation operator and $\rho>0$ is the control weighting. The control and filter spectral factors $D_{c}$ and $D_{f}$ are stable polynomials and are defined, respectively, by

$$
\begin{aligned}
& D_{c} D_{c}^{*}=B B^{*}+A \rho A^{*} \\
& D_{f} D_{f}^{*}=C \psi_{d} C^{*}+A \psi_{n} A^{*}
\end{aligned}
$$

The solution of the optimal regulator problem can now be summarized:

Theorem 1 (Kučera [20], Hunt [21]): The optimal control problem has a solution if and only if

C1) $A$ and $B$ have no unstable common factors.

The optimal regulator polynomials $R$ and $T$ form the unique solution having the property

$$
\left(D_{c}^{*} D_{f}^{*} q^{-g}\right)^{-1} F \quad \text { strictly proper }
$$

of the linear polynomial equations

$$
\begin{aligned}
D_{c}^{*} D_{f}^{*} q^{-g} R+F A & =B^{*} q^{-g} C \psi_{d} C^{*} \\
D_{c}^{*} D_{f}^{*} q^{-g} T-F q^{-k} B & =A^{*} R_{2}
\end{aligned}
$$

where

$$
R_{2}=q^{-g}\left(D_{f} \rho D_{f}^{*}+B \psi_{n} B^{*}\right)
$$

The integer $g>0$ is the smallest integer making (46) and (47) polynomial in $q^{-1}$.

Note that when the common polynomial $F$ is eliminated between (46) and (47), the implied linear equation

$$
A T+q^{-k} B R=D_{f} D_{c}
$$

arises. The conditions under which this single equation may be used to generate the unique optimal regulator are summarized in the following theorem.

Theorem 2 (Hunt and Šebek [37]): The optimal controller polynomials $R$ and $T$ are uniquely determined by the implied equation (49) if and only if:

C2) The subsystem $W_{d}=\frac{C\left(q^{-1}\right)}{A\left(q^{-1}\right)}$ is proper;

C3) $A$ and $B$ are coprime.

The unique solution has the property

$$
A^{-1} R \text { strictly proper. }
$$

A formal proof of these two results can be found in the references [20], [21], and [37]. 
To verify the specialized optimal regulator solution for the muscle moment problem as stated in Section II-A, the following substitutions must be made.

1) $A\left(q^{-1}\right)=\left(1-q^{-1}\right) A_{m}\left(q^{-1}\right)$.

2) $C\left(q^{-1}\right)=1$.

3) $B\left(q^{-1}\right)=B_{m}\left(q^{-1}\right)$.

4) $y(t)=m(t)$.

5) $a(t)=\frac{1}{1-q^{-1}} u(t) \Leftrightarrow u(t)=\left(1-q^{-1}\right) a(t)$.

6) $r(t)=m_{\text {ref }}$.

Note that since the integrator term $1-q^{-1}$ is prespecified in the design, it is considered as part of the plant polynomial $A$.

\section{REFERENCES}

[1] L. A. Benton, L. L. Baker, B. R. Bowman, and R. L. Waters, "Functional electrical stimulation-A practical clinical guide," Rancho Los Amigos Rehabilitation Engineering Center, Downey, CA, Tech. Rep., 1981.

[2] A. Kantrowitz, "Electronic physiological aids," Maimonides Hospital of Brooklyn, NY, Tech. Rep. 1963

[3] A. Kralj and T. Bajd, Functional Electrical Stimulation: Walking and Standing After Spinal Cord Injury. Florida: CRC, 1989.

[4] D. J. Ewins, P. N. Taylor, S. E. Crook, R. T. Liczynski, and I. D. Swain, "Practical low cost sit/stand system for mid-thoracic paraplegics," $J$ Biomed. Eng., vol. 10, pp. 184-188, 1988.

[5] A. J. Mulder, H. B. K. Boom, H. J. Hermens, and G. Zilvold, "Artificialreflex stimulation for FES-induced standing with minimum quadriceps force," Med. and Biol. Eng. and Comput., vol. 28, pp. 483-488, 1990.

[6] D. N. Rushton, N. Donaldson, F. M. D. Barr, V. J. Harper, T. A. Perkins, P. N. Taylor, and A. M. Tromans, "Lumbar anterior root stimulator for lower limb control in paraplegia," in Neuroprosthetics: From Basic Research to Clinical Application, A. Pedotti, M. Ferrarin, J. Quintern, and R. Riener, Eds. New York: Springer-Verlag, 1996.

[7] H. M. Franken, P. H. Veltink, G. Baardman, R. A. Redmeyer, and H. B. K. Boom, "Cycle-by-cycle control of swing phase of paraplegic gait induced by surface electrical stimulation," Med. Biol. Eng. Comput., vol. 33, pp. 440-451, 1995 .

[8] J. Quintern, R. Riener, and S. Rupprecht, "Comparison of simulation and experiments of different closed-loop strategies for FES-Part 2: Experiments in paraplegics," in Proc. 5th Vienna Int. Workshop on Functional Electrostimulation, 1995, pp. 255-258.

[9] P. E. Crago, M. Lemay, and L. Liu, "External control of limb movements involving environmental interactions," in Multiple Muscle Systems: Biomechanics and Movement Organization, J. M. Winters and S. L. Woo, Eds. New York, Springer-Verlag, 1990.

[10] J. T. Mortimer, "Motor prostheses," in Handbook of Physiology -The Nervous System II, J. M. Brookhart, V. B. Mountcastle, V. B. Brooks, and S. R. Geiger, Eds. Bethesda, MD: American Physiological Soc., 1981, pp. $155-187$

[11] G. I. Zahalak, "An overview of muscle modeling," in Neural Prostheses, R. B. Stein, P. H. Peckham, and D. B. Popovic, Eds. Oxford, U.K. Oxford Univ. Press, 1992, pp. 17-57.

[12] N. Donaldson and C.-H. Yu, "FES standing: Control by handle reactions of leg muscle stimulation (CHRELMS)," IEEE Trans. Rehab., vol. 4, Dec. 1996.

[13] N. Donaldson, H. Gollee, K. J. Hunt, J. C. Jarvis, and M. K. N. Kwende, "A radial basis function model of muscle stimulated with irregular inter-pulse intervals," Med. Eng. and Phys., vol. 17, pp. 431-441, Sept. 1995.

[14] H. Gollee and K. J. Hunt, "Nonlinear modeling and control of electrically stimulated muscle: A local model network approach," Int J. Contr., vol. 68, no. 6, pp. 1259-1288, 1997.

[15] H. Gollee, K. J. Hunt, N. Donaldson, and J. C. Jarvis, "Modeling of electrically stimulated muscle," in Multiple Model Approaches to Modeling and Control, R. Murray-Smith and T. A. Johansen, Eds. Taylor and Francis, 1997, Ch. 3, pp. 101-120.

[16] H. J. Chizeck, "Adaptive and nonlinear control methods for neural prostheses," in Neural Prostheses, R. B. Stein, P. H. Peckham, and D. B. Popovic, Eds. Oxford, U.K.: Oxford Univ. Press, 1992, pp. 298-328.

[17] N. Donaldson, F. M. D. Barr, G. F. Phillips, and T. A. Perkins, "Unsupported standing of paraplegics by stimulation of the plantarflexors: Some results from the Wobbler apparatus," in Neuroprosthetics: From Basic Research to Clinical Application, A. Pedotti, M. Ferrarin, J. Quintern, and R. Riener, Eds. New York: Springer-Verlag, 1996.

[18] N. Donaldson, "Practical ankle controllers for unsupported standing in paraplegia," in Proc. Ljubljana FES Conf., 1993, pp. 61-64.
[19] T. J. Bajzek and R. J. Jaeger, "Characterization and control of muscle response to electrical stimulation," Ann. Biomed. Eng., vol. 15, pp. 485-501, 1987.

[20] V. Kučera, Discrete Linear Control: The Polynomial Equation Approach Chichester, U.K.: Wiley, 1979.

[21] K. J. Hunt, "Stochastic optimal control theory with application in selftuning control," in Lecture Notes in Control and Information Sciences, vol. 117. Berlin, Germany: Springer-Verlag, 1989.

[22] D. C. Youla and J. J. Bongiorno, "A feedback theory of two degree of freedom Wiener-Hopf design," IEEE Trans. Automat. Contr., vol. 30 pp. $652-665,1985$

[23] H. Kwakernaak and R. Sivan, Linear Optimal Control Systems. New York: Wiley, 1972.

[24] J. Ježek and K. J. Hunt, "Coupled polynomial equations for LQ control synthesis and an algorithm for solution," Int. J. Contr., vol. 58, no. 5, pp. 1155-1167, 1993.

[25] M. Munih, K. J. Hunt, and N. Donaldson, "LQG control for the ankle joint moment," in Proc. ICMMB Conf., Ljubljana, Slovenia, 1996.

[26] M. Munih, K. J. Hunt, N. Donaldson, and F. M. D. Barr, "LQG moment control in the paraplegic's ankle joint," in Proc. 18th Int. Conf. IEEE Engineering in Medicine and Biology Society, Amsterdam, the Netherlands, 1996 .

[27] K. J. Hunt, M. Munih, and N. Donaldson, "Feedback control of unsupported standing in paraplegia-Part I: Optimal control approach," IEEE Trans. Rehabilitation Eng., vol. 5, pp. 331-340, 1997.

[28] M. Munih, N. Donaldson, K. J. Hunt, and F. M. D. Barr, "Feedback control of unsupported standing in paraplegia-Part II: Experimental results," IEEE Trans. Rehab. Eng., vol. 5, pp. 341-352, 1997.

[29] V. Kučra, Analysis and Design of Discrete Linear Control Systems. Englewood Cliffs, NJ: Prentice-Hall, 1992.

[30] K. J. Åström and B. Wittenmark, Computer Controlled Systems: Theory and Design, 3rd ed. Englewood Cliffs, NJ: Prentice-Hall, 1997.

[31] G. F. Phillips, J. R. Adler, and S. J. G. Taylor, "A portable programmable eight-channel surface stimulator," in Proc. Ljubljana FES Conf., 1993, pp. $166-168$.

[32] W. K. Durfee and K. E. Maclean, "Methods of estimating the isometric recruitment curve of electrically stimulated muscle," Trans. IEEE Biomed. Eng., vol. 36, pp. 654-667, 1989.

[33] L. Ljung and T. Söderström, Theory and Practice of Recursive Identification. London, U.K.: MIT Press, 1983.

[34] L. Ljung, System Identification-Theory for the User. Englewood Cliffs, NJ: Prentice-Hall, 1987.

[35] K. J. Hunt, M. Munih, N. Donaldson, and F. M. D. Barr, "Investigation of the Hammerstein hypothesis in the modeling of electrically stimulated muscle," IEEE Trans. Biomedical Eng., to be published.

[36] A. Ishida and S. Miyazaki, "Maximum likelihood identification of a posture control system," IEEE Trans. Biomed. Eng., vol. 34, pp. 1-5, 1987.

[37] K. J. Hunt and M. Šebek, "Implied polynomial matrix equations in multivariable stochastic optimal control," Automatica, vol. 27, pp. 395-398, 1991

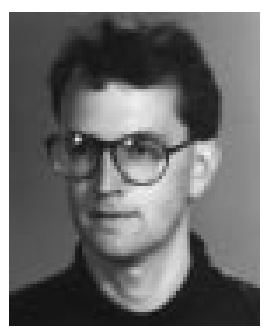

Kenneth J. Hunt (S'87-M'87) received the B.Sc. degree (first class hons.) in electrical and electronic engineering in 1984 and the Ph.D. degree in control theory in 1987, both from the University of Strathclyde, Glasgow, Scotland.

From 1987 to 1989 he was a Research Scientist with BBN Systems and Technologies (the European division of Bolt, Beranek and Newman Inc.). From 1989 to 1992 he held a Royal Society of Edinburgh Personal Research Fellowship, and during that period was with the Department of Mechanical Engineering at the University of Glasgow. He joined DaimlerBenz in November 1992 and was a Research Scientist and Project Leader with Daimler-Benz Systems Technology Research Berlin, part of DaimlerBenz corporate research, until 1997. He is currently a Professor of Mechanical Engineering in the Centre for Systems and Control at the University of Glasgow, Scotland. He is also a Visiting Professor at the Department of Automatic Control and Systems Engineering at the University of Sheffield.

In August 1992, Dr. Hunt was Local Arrangements Chair at the IEEE International Symposium on Intelligent Control in Glasgow. A Chartered Engineer, he is a member of the Institution of Electrical Engineers and contributes to the IFAC Technical Committee on Adaptive Systems. He is chairman of IFAC's recently formed Technical Committee on Fuzzy and Neural Systems and is an Associate Editor of the IEEE Transactions ON Control Systems TeChNOLOGy. 


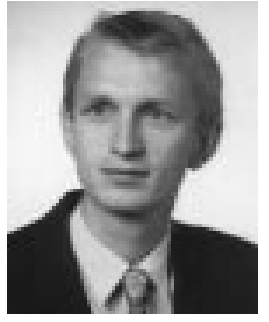

Marko Munih (M'88) received the B.Sc. and M.Sc. degrees in electrical engineering from the University of Ljubljana, Slovenia, in 1986 and 1989, respectively. He received the D.Sc. degree in electrical engineering in 1993 for work on controlling muscle activity with respect to the femur and tibia bone loading.

In 1989 he was appointed as a Teaching Assistant at the Faculty of Electrical Engineering. From 1995 to 1996 he was a Research Assistant with the Implanted Devices Group in the Department of Medical Physics and Bioengineering at University College London, where he did research on unsupported standing of paraplegics and development of the sensory amplifier. Currently, he is a faculty member at the Faculty of Electrical Engineering, University of Ljubljana, Slovenia. His research interests include functional electrical stimulation of paraplegic lower extremities with surface electrode systems, including measurement, control, biomechanics and electrical circuits.

Dr. Munih is member of IFMBE, IFESS, and IFAC and serves as an international referee for several journals in the Biomedical Engineering field.

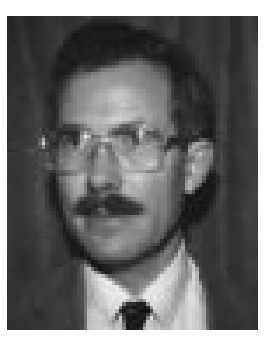

Nick Donaldson studied engineering at Cambridge University. He received the Ph.D. degree from London University, U.K.

After time spent designing integrated circuits at the General Electric Company, he joined the Medical Research Council Neurological Prostheses Unit under the direction of Giles Brindley in 1976. Since then he has worked on the use of surgically implanted devices for restoring paralyzed limb function. Since 1992, he has been Head of the Implanted Devices Group at University College London, which has a close collaboration with the Spinal Injuries Units at the Royal National Orthopaedic Hospital and Salisbury Hospital in England. His research interests include implant technology, implanted orthopaedic instrumentation, the biomechanics of standing, biomechanical instrumentation, nerve and nerve root stimulation, feedback control in FES, and use of ENG signals in control.

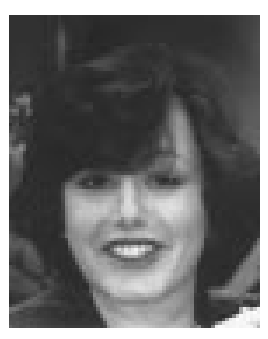

Fiona M. D. Barr received the diploma from the Bristol School of Physiotherapy and an M.Sc. degree at Kings College, London, U.K. She is currently preparing a Ph.D. thesis on the quantification of spasticity.

She is a Research Physiotherapist at the Royal National Orthopaedic Hospital, Stanmore, U.K., with much experience in the application of FES to patients with spinal cord injuries. 